\title{
Spin precession due to a non-Abelian spin-orbit gauge field
}

\author{
Jyh-Shinn Yang, ${ }^{1}$ Xiao-Gang He, ${ }^{2}$ Son-Hsien Chen, ${ }^{2}$ and Ching-Ray Chang ${ }^{2, *}$ \\ ${ }^{1}$ Institute of Optoelectronic Science, National Taiwan Ocean University, Keelung 202, Taiwan \\ ${ }^{2}$ Department of Physics and Center for Theoretical Sciences, National Taiwan University, Taipei 10617, Taiwan
}

(Received 5 February 2008; revised manuscript received 3 June 2008; published 20 August 2008)

\begin{abstract}
We study the spatial behavior of spin precession for traversing electrons in a two-dimensional system with both the Rashba and Dresselhaus spin-orbit (SO) couplings. Treating the two SO coupling as non-Abelian SO gauges and performing the unitary gauge transformation for the Hamiltonian, the effect of SO coupling is exactly represented by a spin-rotation operator providing a convenient framework for studying the property of ballistic spin transport. We derive the analytical expression for the spin configuration and demonstrate a classical analog of spin precession. The present approach provides a powerful means (e.g., the spin-rotation axis, the precession angle, and the cone angle) allowing concrete description of the local spin orientation. In particular, the spatial features such as the specific transport path with null spin precession and the special contour with complete cycles of spin precession can be easily identified.
\end{abstract}

DOI: 10.1103/PhysRevB.78.085312

PACS number(s): 72.25.Dc, 71.70.Ej, 73.23.-b

\section{INTRODUCTION}

The spin-orbit (SO) coupling attracts much attention not only for its profound fundamental spin physics but also for its wide applications in spintronic devices. ${ }^{1,2}$ The SO coupling is an intrinsic effect and makes the spin of electrons couple with their orbital motion, thereby providing a promising means to manipulate the electron spin in semiconductor ${ }^{3}$ and metallic nanostructures ${ }^{4}$ without an external magnetic field. The dominant SO couplings relevant in planar semiconductor heterostructures are the Rashba and Dresselhaus effects. The former stems from the structure inversion asymmetry (SIA), ${ }^{5}$ which can be controlled by the gate voltage, ${ }^{6,7}$ while the latter is due to the bulk inversion asymmetry (BIA), ${ }^{8}$ inducing the SO coupling with strength being material specific. The SO coupling causes the spinstate splitting acting as an effective magnetic field (EMF) (Ref. 9) about which the spin of transverse electrons undergoes precession within a two-dimensional electron gas (2DEG). This unique property due to the inversion asymmetry in 2DEG channels opens the possibility of spin-based devices, e.g., spin filter, ${ }^{10,11}$ spin-field-effect transistor, ${ }^{7,12,13}$ and spin pumping. ${ }^{14}$ To date, the real device remains elusive. Thus, deeply understanding and properly controlling the behavior of spin precession due to the SIA and/or BIA effects are crucial to the practical realization of such devices. ${ }^{15}$

In general, the EMF depends on the travel direction of electrons while in the two special cases of [001] RashbaDresselhaus model with equal Rashba and Dresselhaus coupling constants and the [110] Dresselhaus model, it is independent of the travel direction of electron. ${ }^{16,17}$ As a result (in such two cases), the precession angle of spin depends only on the distance traveled along a specific spatial direction. Accordingly, the electron precesses as a helixlike pattern called persistent spin helix (PSH). ${ }^{16,17}$ In addition, the pattern of PSH is persistent against any momentum-dependent (but spin-independent) scatterings. The phenomenon can also be rationalized in terms of a $S U(2)$ spin-rotation symmetry. Moreover, it has been shown that the Elliot-Yafet spin-flip mechanism or the D'yakonov-Perel' spin-relaxation mecha- nism can be suppressed substantially in the above system ensuring the long lifetime of spin. Thus, the utilization of this persistency without ballistic conditions required makes the high-performance transistor possible. . $^{12,13}$

Earlier study by Jin et al..$^{18}$ showed that the SO coupling including Rashba and Dresselhaus terms can be regarded as $S U(2)$ gauge potential leading to a force acting on the spin and spin current induced by the Yang-Mills fields. Independently, Hatano et al. ${ }^{11}$ treated the Rashba SO (RSO) coupling as non-Abelian $S U(2)$ gauge, which imposes spin-dependent phases on the traveling electron. By neglecting the Dresselhaus spin-orbit (DSO) coupling and further adjusting the strength of the RSO coupling and the magnetic field, they achieve ideal spin-filtering rings capable of producing one type of spin-polarized electron currents. Chen and Chang ${ }^{19}$ recently utilized the non-Abelian SO gauge for the coexistence of RSO and DSO couplings to demonstrate that the PSH can be easily understood from the perspective of gauge transformation and found that the quantum square ring functions as a versatile device with four spin states.

In this paper, we shall focus on the spin-precession properties of conduction electrons in the RSO and DSO coupling system. There have been indeed similar studies on the issue; $;, 15$ the essential difference here is that the SO coupling can be neatly represented by a spin-rotation operator by gauge transformation and then we can study the subject of spin transport along the straight path without the dynamic equation involving inflicting lengthy algebra. Based on this operator, we derive an analytical formula to describe the spin configuration in more transparency and explicitly specify the spatial features of spin configuration such as the path with null spin precession and the contour with complete cycles of spin precession. The present approach also provides a natural scheme for investigating the subject of spin interference in one-dimensional ballistic polygon loops. The remainder of this paper is organized as follows. In Sec. II we describe the theoretical approach to perform the gauge transformation and derive the spin-rotation operator due to the SO coupling. In Sec. III the expectation value for the injected spin and the heuristic analog of classical spins for spin precession are proposed and then followed by the numerical results for the 
spin configuration using a pictorial interpretation with a highlight of special contours and paths. Finally discussion and conclusion are in turn made.

\section{THEORY}

Consider an electron with a definite spin perfectly injected via an ideal point contact-setting on the origin $(0,0)$ - into an inversion-asymmetric 2DEG where both the RSO and DSO couplings are present. The 2DEG is assumed to be semi-infinite so that the boundary effect is out of consideration. Setting the growth direction of the 2DEG layer to be [001] and the $x$ and $y$ axes to be [100] and [010], respectively, the Hamiltonian in the presence of the SO coupling for a single electron of effective mass $m$ is given by

$$
H=\frac{1}{2 m}\left(p_{x}^{2}+p_{y}^{2}\right)+\frac{\alpha}{\hbar}\left(p_{y} \sigma_{x}-p_{x} \sigma_{y}\right)+\frac{\beta}{\hbar}\left(p_{x} \sigma_{x}-p_{y} \sigma_{y}\right),
$$

where $p_{x, y}$ is the momentum operator of electron within the 2DEG, $\alpha$ and $\beta$ are the strengths of the RSO and DSO couplings, respectively, and $\sigma_{x, y}$ are known as the Pauli spin matrices. The key to the analysis below is to make the linear term in Eq. (1) disappear. To this end, the following SO gauge is utilized: ${ }^{18,19}$

$$
\vec{A}_{\mathrm{SO}}=\left(A_{x}, A_{y}\right) \equiv \frac{m c}{e \hbar}\left(\alpha \sigma_{y}-\beta \sigma_{x},-\alpha \sigma_{x}+\beta \sigma_{y}\right) .
$$

Performing the unitary transformation to the Hamiltonian $H \rightarrow U^{\dagger} H U \equiv H_{0}$, where $U=\exp \left[(i e / \hbar c) \vec{A}_{\mathrm{SO}} \cdot \vec{r}\right]$, the SO coupled Hamiltonian in Eq. (1) is thus mapped to that of the free Fermi gas,

$$
H_{0}=\frac{1}{2 m}\left(\vec{p}-\frac{e}{c} \vec{A}_{\mathrm{SO}}\right)^{2}-V I_{s},
$$

with the constant potential $V=\left(m / h^{2}\right)\left(\alpha^{2}+\beta^{2}\right)$ and $I_{s}$ is the $2 \times 2$ unit matrix. Note that the components of gauge $\vec{A}_{\text {SO }}$ do not commute with each other,

$$
\left[A_{\mathrm{SO}, x}, A_{\mathrm{SO}, y}\right]=2 i\left(\frac{m c}{e \hbar}\right)^{2}\left(\alpha^{2}-\beta^{2}\right) \sigma_{z} .
$$

Thus, we have a non-Abelian gauge field. With this gauge field, we may construct the local transformation operator $U(\vec{r})=\exp \left[(i e / \hbar c) \int_{c} \vec{A}_{\mathrm{so}}\left(\vec{r}^{\prime}\right) \cdot d \vec{r}^{\prime}\right]$ with $\vec{r}=(x, y)$. In general, $U(\vec{r})$ depends on the actual integration path $c$. Nevertheless, in the case of uniform SO coupling of both Rashba and Dresselhaus types, since $\vec{A}_{\mathrm{SO}}$ is independent of position $\vec{r}$, we have $\nabla \times \vec{A}_{\mathrm{SO}}=0$ so that $\int_{c} \vec{A}_{\mathrm{SO}}\left(\vec{r}^{\prime}\right) \cdot d \vec{r}^{\prime}=\vec{A}_{\mathrm{SO}} \cdot \vec{r}$. This yields the expression

$$
U(\vec{r})=\exp \left[\frac{i e}{\hbar c}\left(\vec{A}_{\mathrm{SO}} \cdot \vec{r}\right)\right],
$$

with the unitary property $U(\vec{r}) U^{+}(\vec{r})=I_{s}$ ensured by the Hermitian $\vec{A}_{\mathrm{SO}}^{+}=\vec{A}_{\mathrm{SO}}$ from the definition (2).
We note that Eq. (3) differs from the free-electron gas with Hamiltonian $H_{0}$ only by a gauge $(e / c) \vec{A}_{\mathrm{SO}}$. If the following commutation relation is satisfied:

$$
\begin{aligned}
& {\left[A_{\mathrm{SO}, x} x+A_{\mathrm{SO}, y} y, A_{\mathrm{SO}, x} x_{0}+A_{\mathrm{SO}, y} y_{0}\right]} \\
& \quad=2 i\left(\frac{m c}{e \hbar}\right)^{2}\left(\alpha^{2}-\beta^{2}\right)\left(x y_{0}-x_{0} y\right) \sigma_{z}=0,
\end{aligned}
$$

for any $x_{0}$ and $y_{0}$, we obtain the gauge transformation

$$
U(\vec{r}) H_{0} U(\vec{r})^{+}=H .
$$

Consider first an injected electron with initial spin state $\chi_{\text {inj }}$ in system $H_{0}$. Clearly, without any spin-dependent mechanisms, this electron shall retain its spin state as it traverses the sample. Now, consider $\vec{A}_{\mathrm{SO}}$, corresponding to the turning on $U(\vec{r})$ so that the electron wave function in the SO coupling system undergoes a gauge transformation of $U(\vec{r})$. If the electron travels along the straight-line path-i.e., the relation (6) is met - the spin polarization of the traversing electron varies spatially according to

$$
U(\vec{r}) \chi_{\mathrm{inj}}=\exp \left[\frac{i e}{\hbar c}\left(\vec{A}_{\mathrm{SO}} \cdot \vec{r}\right)\right] \chi_{\mathrm{inj}} \equiv \exp [i \eta C] \chi_{\mathrm{inj}} .
$$

Here $\eta=\left(m / \hbar^{2}\right)\left[\left(\alpha^{2}+\beta^{2}\right)\left(x^{2}+y^{2}\right)+4 \alpha \beta x y\right]^{1 / 2}$ and $C$ is a 2 $\times 2$ matrix introduced for later convenience with elements $C_{11}=C_{22}=0$ and $C_{12}=C_{21}^{*}=-i \exp (i \xi)$, where $\xi$ is defined by $\cos \xi=(\alpha x+\beta y) / b$ and $\sin \xi=-(\alpha y+\beta x) / b$ with $b$ $=\left(\hbar^{2} / m\right) \eta$. As shown below, the symbols $\eta$ and $\xi$ specify the two directional angles of quantum-mechanical rotation operators for spin $1 / 2$.

Our purpose now is to make the operator $U(\vec{r})$ tractable and expressible in usual $2 \times 2$ spin matrices. With matrix $C$ being diagonalizable, it is advisable to decompose the matrix $C$ in terms of Pauli matrix

$$
C=V^{+} u V
$$

where

$$
u=\sigma_{y}=\left(\begin{array}{cc}
0 & -i \\
i & 0
\end{array}\right)
$$

and

$$
V=\left(\begin{array}{cc}
1 & 0 \\
0 & e^{i \xi}
\end{array}\right) .
$$

Note that matrices $C$ and $u$ have identical eigenvalues \pm 1 . Since the matrix $u$ is Hermitian, it can be diagonalized by a unitary transformation $D u D^{\dagger}$, where $D$ is the matrix of eigenvectors of $u$. After some algebra, the unitary matrix $D$ is easily obtained as

$$
D=\frac{1}{\sqrt{2}}\left(\begin{array}{cc}
i & 1 \\
-i & 1
\end{array}\right) .
$$

Immediately the diagonal matrix $D u D^{\dagger}$ is just the Pauli matrix $\sigma_{z}$. With the above results, the unitary equivalent matrices $u$ and $D \sigma_{z} D^{\dagger}$ should have identical spectra. ${ }^{20}$ In other words, the matrix $C$ in Eq. (9) can be rewritten as 


$$
C=\left(V^{+} D\right) \sigma_{z} D^{+} V \equiv L^{+} \sigma_{z} L,
$$

where the matrix $L\left(=D^{\dagger} V\right)$ is

$$
L=\frac{1}{\sqrt{2}}\left(\begin{array}{cc}
i & e^{i \xi} \\
-i & e^{i \xi}
\end{array}\right) .
$$

With $L^{\dagger}=L^{-1}$, the unitary operator $U(\vec{r})$ readily becomes

$$
U(\vec{r})=\exp \left[i L^{+}\left(\eta \sigma_{z}\right) L\right]=L^{+} \exp \left(i \eta \sigma_{z}\right) L .
$$

Now we can expand the exponential of the matrix $i \eta \sigma_{z}$ as a power series. Using the properties of Pauli matrices $\sigma_{i}^{2}=I_{s}$ and grouping together the coefficients of $I_{s}$ and $\sigma_{z}$ lead to the expression in $2 \times 2$ form,

$$
\exp \left(i \eta \sigma_{z}\right)=\left(\begin{array}{cc}
e^{i \eta} & 0 \\
0 & e^{-i \eta}
\end{array}\right) .
$$

Substituting Eq. (15) back into Eq. (14) and performing two successive operations of matrix product, the closed form of unitary operator $U(\vec{r})$ is readily obtained,

$$
U(\vec{r})=\left(\begin{array}{cc}
\cos \eta & \sin \eta e^{i \xi} \\
-\sin \eta e^{-i \xi} & \cos \eta
\end{array}\right) .
$$

By comparison with the rotation operator for spin $1 / 2,{ }^{20}$ the operator $U(\vec{r})$ clearly shows that the spin of traversing electron undergoes a rotation about the axis $\hat{n}$ $=-(\sin \xi, \cos \xi, 0)$ by a (precession) angle $2 \eta$. In this sense, Eq. (16) gives a vivid physical picture of spin precession due to both SIA and BIA effects. The essence of the above unitary transformation is that we choose a space-dependent spin coordinate, following the spin precession, in which the spin-up direction is kept fixed everywhere in the rotating frame but the other two spin directions are dependent on the space position $\vec{r}$. Since the spin precesses about the EMF due to the SO coupling, the above axis $\hat{n}$ would align with the direction of EMFs that provides the relevant quantum axis of spin (see explanation below). ${ }^{9}$ Note that the axes $\hat{n}$ due to Eq. (16) are in accord with those directly obtained from Eq. (5) for the special cases with $\alpha \beta=0$ or \pm 1 . Obviously, Eq. (16) demonstrates that the net spin precession in the $x y$ plane for traversing electrons depends only on the net displacement $\vec{r}$ relative to the injected point and is independent of any other property of the electron's trajectory. Also, the oscillatory dependence of $U(\vec{r})$ on $x$ and $y$ that appears for general $\alpha$ and $\beta$ is responsible for the spin-dependent phenomena such as PSH and spin interference.

\section{RESULTS AND DISCUSSION}

We now demonstrate the spatial features of spin precession due to different mechanisms of inversion asymmetry by employing the present strategy without inflicting lengthy algebra. We assume that the electron possesses a conserved wave vector and is free to move in the 2DEG plane along any crystallographic direction. To keep things simple, the case with $\alpha \geq 0$ and $\beta \geq 0$ is only considered here and the result for other cases can be inferred by symmetry.

Using Eq. (16), we have vanishing off-diagonal elements if $\sin \eta=0$ or $\eta=n \pi$ with $n$ being an integer. This means that

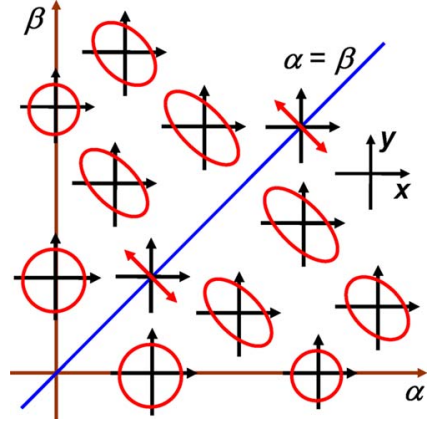

FIG. 1. (Color online) The $x-y$ contour in the $\alpha-\beta$ plane where the spins all return to their original orientation. Note that the contours in other regimes can be inferred by the mirror symmetry with respect to the $\alpha$ or $\beta$ axis and the point symmetry under replacement $(\alpha, \beta) \rightarrow(-\alpha,-\beta)$.

the spin undergoes just the complete cycles of spin precession as the precession angle is $2 n \pi$. Thus, the spin of traversing electron all return exactly to the original orientation after arriving at these specific positions. Putting $\eta=n \pi$ leads to

$$
\left(\alpha^{2}+\beta^{2}\right)\left(x^{2}+y^{2}\right)+4 \alpha \beta x y=\frac{n^{2} \pi^{2} \hbar^{4}}{m^{2}} .
$$

Then transforming the coordinates $x=(p-q) / \sqrt{2}$ and $y=(p$ $+q) / \sqrt{2}$ and introducing the reduced one $\tilde{p}=p / R_{0}$ and $\tilde{q}$ $=q / R_{0}$ with $R_{0}=\pi \hbar^{2} / m\left(\alpha^{2}+\beta^{2}\right)^{1 / 2}$, we arrive at

$$
\frac{\tilde{p}^{2}}{(\alpha-\beta)^{2}}+\frac{\widetilde{q}^{2}}{(\alpha+\beta)^{2}}=\frac{n^{2}\left(\alpha^{2}+\beta^{2}\right)}{\left(\alpha^{2}-\beta^{2}\right)^{2}} .
$$

Obviously, Eq. (17) indicates that depending on the coupling ratio of $\alpha / \beta$, the special contour may be a family of parallel lines, concentric circles, or ellipses for the cases with $\alpha / \beta$ $= \pm 1, \alpha \beta=0$, or others, respectively (Fig. 1). Note that the phase diagram exhibits a mirror symmetry with respect to the $\alpha$ or $\beta$ axis and a point symmetry under replacement $(\alpha, \beta) \rightarrow(-\alpha,-\beta)$. Remarkably, the short axes of ellipses all align along either $\pm[110]$ or $\pm[1-10]$ directions due to the high spin-precession rate (with the travel distance) along the four specific paths on which the EMFs due to the BIA and SIA effects are parallel to each other as explained below.

In order to study the spin configuration, we seek for the expectation value of the spin components $S_{x}=\left\langle\sigma_{x}\right\rangle, S_{y}=\left\langle\sigma_{y}\right\rangle$, and $S_{z}=\left\langle\sigma_{z}\right\rangle$ along the three principal directions disregarding the factor $\hbar / 2$. Straightforward mathematics yields

$$
\begin{aligned}
\left\langle\sigma_{x}\right\rangle= & \sin \theta_{s}\left\{\sin \left(\phi_{s}+\xi\right) \sin \xi+\cos (2 \eta)\left[\cos \phi_{s}-\sin \left(\phi_{s}\right.\right.\right. \\
& +\xi) \sin \xi]\}-\cos \theta_{s} \sin (2 \eta) \cos \xi \\
\left\langle\sigma_{y}\right\rangle= & \sin \theta_{s}\left\{\sin \left(\phi_{s}+\xi\right) \cos \xi+\cos (2 \eta)\left[\sin \phi_{s}-\sin \left(\phi_{s}\right.\right.\right. \\
& +\xi) \cos \xi]\}+\cos \theta_{s} \sin (2 \eta) \sin \xi \\
\left\langle\sigma_{z}\right\rangle & =\sin \theta_{s} \sin (2 \eta) \cos \left(\phi_{s}+\xi\right)+\cos \theta_{s} \cos (2 \eta),
\end{aligned}
$$

for an injected spin with arbitrary polarization $\chi_{\text {inj }}$ $=\left\langle\cos \left(\theta_{s} / 2\right), \sin \left(\theta_{s} / 2\right) \exp \left(i \phi_{s}\right)\right\rangle^{T}$, where $\theta_{s}$ and $\phi_{s}$ are the 
polar and azimuthal angles, respectively. ${ }^{20}$ It is worthy of note that for the injected spin pointing in the 2DEG plane, the present expressions (18a)-(18c) are exactly reduced to Eq. (5) in Ref. 3. Analyzing the origin of the terms in Eqs. (18a)-(18c) further gives the physical insight into the feature of spin precession. Obviously, the last terms are due to the out-of-plane component of injected spin and the others are due to the in-plane one. The first term in the brace, namely, the component of the injected spin along the spin-rotation axis, is always kept constant along the straight-line path while the other terms are sinusoidal functions of $\eta$ or $x$ and $y$-exactly accounting for the spin precession. Thus, Eqs. (18a)-(18c) significantly demonstrate that the spatial behavior of spin precession under the SO coupling can be treated as a precession of a classical spin with constant velocity traveling through the uniform in-plane magnetic field. In other words (as moving with the traversing spin), we see that the spin continuously precesses on a cone around the effective field where the cone angle is the smaller one of $\mid \phi_{S}+\xi$ $+\pi / 2 \mid$ or $\left|\phi_{s}+\xi-3 \pi / 2\right|$. One can easily check that the result (17) can be obtained again when putting $\eta=n \pi$ in Eqs. (18a)-(18c). Moreover, the spin direction always keeps unchanged as $\theta_{s}=\pi / 2$ and $\phi_{s}= \pm \pi / 2-\xi$; namely, spin injection into eigenspinor states ${ }^{12,17}$ leads to the null spin precession. This fact justifies that the spin-rotation axis specified by the above unitary matrix $U(\vec{r})$ provides one of the quantum axes of local spin.

As mentioned above, we remark that there are always two crossed straight lines in the pattern of spin precession except for the special cases with $\alpha / \beta= \pm 1$; one refers to the pair of straight paths with spin precession having a maximum cone angle at $\phi_{1}=\phi_{u}$ or $\phi_{u}-\pi$ with $\phi_{u}=\tan ^{-1}[(\alpha p+\beta) /(\alpha+p \beta)]$ and $p=\tan \left(\phi_{s}\right)$ and the other one is that with null spin precession at $\phi_{2}=\phi_{u} \pm \pi / 2$ (see Figs. 2 and 3). Note that the above crossed lines become the Greek cross coincidentally for the case with $\alpha \beta=0$ and $\phi_{s}=0$. Interestingly, the crossed straight lines rotate $\pi / 2-2 \phi_{s}$ counterclockwise as the coupling ratio of $\beta / \alpha$ increases from zero to infinity. Next, we shall show that the spatial distribution of spin orientation along other paths can be satisfactorily described by ways of the relevant spin-rotation axis and cone angle. A full twodimensional spin configuration is plotted pictorially for the convenience of reference and complements of Ref. 3. As an explicit illustration, we assume that the electron with spin polarization along the $x$ axis (i.e., $\theta_{s}=\pi / 2$ and $\phi_{s}=0$ ) is injected at an angle $\phi$ relative to the $x$ axis.

Now we start with the pure Rashba case $(\beta=0)$, i.e., only the SIA effect present. Using Eq. (8), we easily found that the spin-precession angle is $2 m \alpha r / \hbar^{2}$, which is proportional to the travel distance as well as the coupling strength. With $\xi=-\phi$, the spin-rotation axis is $(y / r,-x / r, 0)-$ or $\hat{p} \times \hat{z}$-always perpendicular to the travel path of traversing electron and spanning an angle $\phi-\pi / 2$ with the $x$ axis. This fact implies the well-known feature of the Rashba field, which is circularly polarized. Such an EMF manifests that the RSO coupling is independent of the crystallographic direction and is invariant under rotation about the $z$ axis of the 2DEG plane. The spatial behavior of the Rashba spin precession (RSP) with space (rather than time) shown in Fig. 2(a) is clearly observed where the special contours, a family of
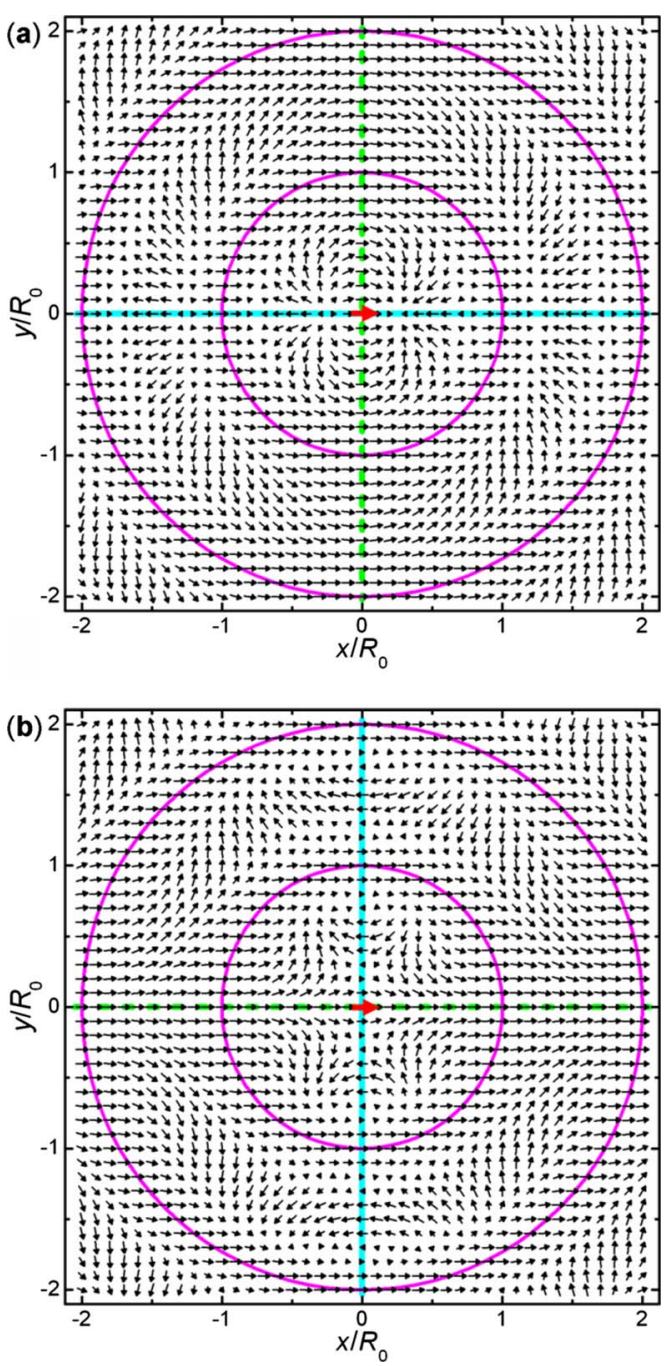

FIG. 2. (Color online) Spin precession due to the (a) pure Rashba and (b) pure Dresselhaus spin-orbit couplings. The length unit (a) $R_{0}=\pi \hbar^{2} / m \alpha$ or (b) $\pi \hbar^{2} / m \beta$. The injected spin, shown by the (red) bold arrow on $(0,0)$, is polarized along [100]. The circles (magenta) denote the contours where the spins all return to their original orientation. The crossed lines refer to the paths with null spin precession (dashed, green) or the paths with spin precession having maximum cone angles (solid, cyan).

concentric circles, and two crossed straight lines are highlighted. The RSP along the transport path generally behaves simply like a pendulum swinging about the Rashba field direction resulting from the spin-rotation axis perpendicular to the travel direction. While on the $\phi_{2}$ path-the $\pm y$ axes in the present case - the spin-rotation axis is parallel to the polarization of injected spin; thus, the cone angle becomes zero and the null spin precession should appear. As the travel path gradually deviates from the $\phi_{2}$ path, the cone angle increases linearly and the component of local spin along the spinrotation axis decreases accordingly except on the special contours [the circles in Fig. 2(a)]. Finally, as the travel path is along the $\phi_{1}$ path or the $\pm x$ axes, the spin exhibits an upright precession with a cone angle $\pi / 2$. Note that the prominent contours where the spins all return to its original 

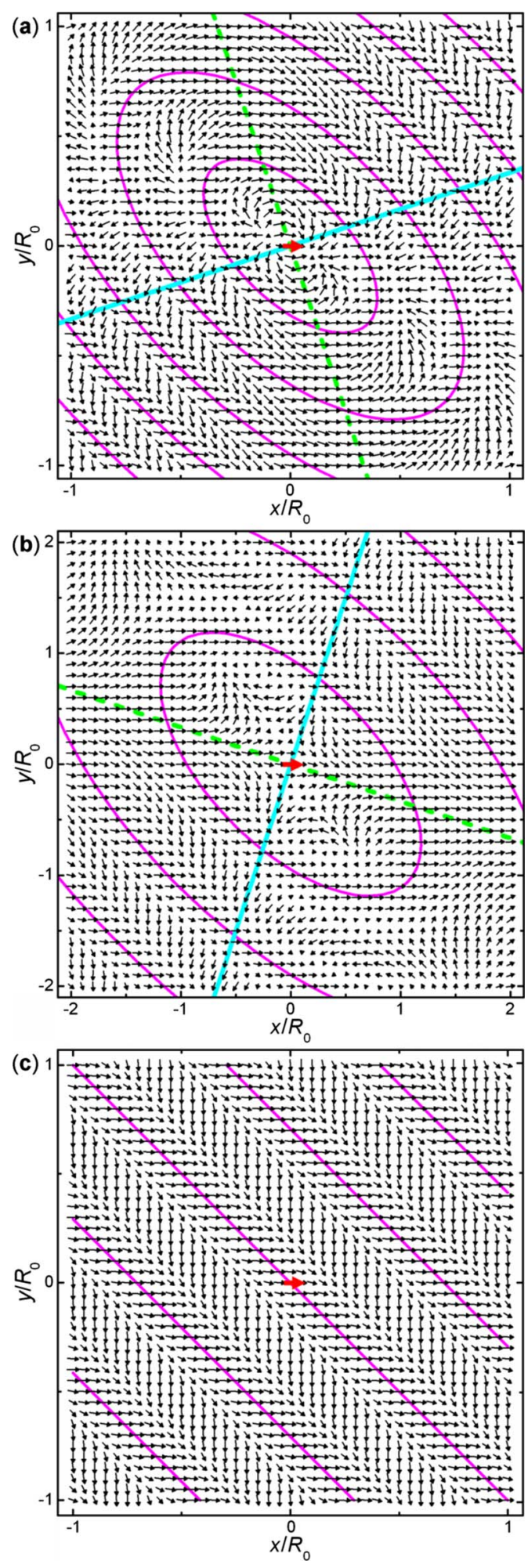

FIG. 3. (Color online) Spin precession due to the combined Rashba and Dresselhaus spin-orbit couplings with the coupling ratios (a) $\alpha / \beta=3$, (b) $1 / 3$, and (c) 1 . The length unit $R_{0}=\pi \hbar^{2} / m \beta$. The injected spin, shown by the (red) bold arrow on $(0,0)$, is polarized along [100]. The ellipses or lines (magenta) denote the contours where the spins all return to its original orientation. The crossed lines refer to the paths with null spin precession (dashed, green) or the paths with spin precession having maximum cone angles (solid, cyan). orientation are a family of concentric circles due to the rotation symmetry of the Rashba fields.

In the case with only Dresselhaus coupling $(\alpha=0)$, the spin-precession angle is found to be $2 m \beta r / \hbar^{2}$, suggesting the directional independence of the coupling strength, similar to the Rashba case. With $\xi=\phi-\pi / 2$, the spin-rotation axis is $(x / r,-y / r, 0)$ spanning an angle $-\phi$ with the $x$ axis; the direction of effective magnetic fields (EMFs) is no more perpendicular to the electron's momentum except the symmetrypreferred paths along the directions $\phi= \pm 45^{\circ}\left( \pm 135^{\circ}\right)$. Thus, the injected spin encounters another type of EMFs and, hence, the spatial behavior of Dresselhaus spin precession (DSP) on the general path is quite different from the Rashba one [see Fig. 2(b)] as expected. While for $\phi= \pm 45^{\circ}$ $\left( \pm 135^{\circ}\right)$, the DSP exhibits a similar spatial behavior with the RSP. In addition, the rotational invariance about the $z$ axis, contrary to the Rashba case, is broken and the crystallographic direction dependence hence comes into play. Although the DSP appears to be more complicated than the RSP, the main feature of position-dependent spin orientation can be still understood in terms of the relevant spin-rotation axis and cone angle. It is interesting that although the Rashba and Dresselhaus couplings behave quite differently, the highlighted curves in the pattern of DSP look the same as the RSP with the essential difference being the orientations of the $\phi_{1}$ and $\phi_{2}$ paths. The occurrence of the same circular contour with precession angles of $2 n \pi$ is reasonably expected because: (i) the two types of effective-field strengths exhibit the crystallographic direction independence and (ii) the spin-precession rate under the fixed EMF has nothing to do with the angle of injected spin polarization relative to its rotation axis (just like the precession period of a spinning top) irrespective of the tilting of its rotation axis.

In the case with coexisted Rashba and Dresselhaus couplings, the spin undergoes precession about the sum of EMFs due to the two couplings. The spin-rotation axes corresponding to the two couplings are generally not collinear-except those along the symmetry-preferred paths. As a result, the spin texture cannot be easily determined without specific knowledge of the strengths of bulk inversion asymmetry (BIA) and structure inversion asymmetry (SIA) effects. However, for the cases dominated by the SIA or BIA effects, the configuration of spin orientation, as expected, should exhibit the RSP-type or the DSP-type behavior as shown in Figs. 3(a) and 3(b). Notably, the special contours (see the curves) become ellipses and their short axes all align along the [110] direction due to the high spin-precession rate along the two paths stemming from the enhancement of the Rashba and Dresselhaus fields. Moreover, the two crossed straight lines still appear and become tilted toward the diagonal direction. We remark that the small variation in spatial distribution of spin orientation occurs around the point where the $\phi_{2}$ path intersects the special contour. The property can benefit us to enable the high efficiency of the spin device based on the coherent spin rotation with tolerance of the uncertainty in the rotation angle resulting from the uncertainties in the detected position, the coupling ratio, and the randomization of spin states caused by the spin-related scattering. When the coupling constants in the Rashba and Dresselhaus terms are equal $(\alpha=\beta)$, we obtain that the spin-precession 
angle is $\left(2^{3 / 2} \alpha m / \hbar^{2}\right)|x+y|$ and that the spin-rotation axis is constant, i.e., $[1-10]$ for $x+y>0$ and $[-110]$ otherwise. Therefore, the fixed direction of rotation axis on either side of the $135^{\circ}\left(-45^{\circ}\right)$ axis reasonably makes the spin-precession angle to be merely proportional to the net displacement along the direction [110]_-disregarding the travel path and the initial spin orientation. As a result, the special pattern of spin precession, called the persistent spin helix (PSH), ${ }^{16,17}$ is developed as shown in Fig. 3(c). This pattern exhibits the translation invariance with a period of spin-precession length $\pi \hbar^{2} / 2 m \alpha$ or the distance between the adjacent contours with complete spin-precession cycles. A similar pattern of PSH can also be observed in the case with $\alpha=-\beta$ (not shown here).

Finally, we demonstrate the convenience of the present scheme to study the spin interference in one-dimensional ballistic polygon loops. We consider the tilted square ring with a side length $\ell$ (see Fig. 1 in Ref. 19) rotated by an angle $\theta$ clockwise with respect to the $x$ axis; the lower-left and the upper-right corners are in contact with two ideal leads. Assume here that the spin-orbit (SO) coupling exists only within the loop but it is absent in the two leads. We focus on the SO coupling induced phase and neglect the effect of external magnetic fields since the latter just creates an additional spin-independent phase of the same sign for both up and down spins. Suppose now that an incident spin $\psi_{\text {inc }}$ from the left lead is split into a pair of partial waves at the lower-left corner and then each of them follows the bottom-right path anticlockwise (called path I) or the left-top path clockwise (called path II). They finally merge at the upper-right corner and enter into the right lead giving rise to the spin interference. Using the above spin-rotation operator $U$, the outgoing spin $\psi_{\mathrm{I}, \mathrm{II}}$ along the path I (II) can be conveniently described by the successive matrix product of $U$ along the travel path, i.e., $\psi_{\mathrm{I}, \mathrm{II}}=U_{\mathrm{I}, \mathrm{II}} \psi_{\text {inc }}$, where $U_{\mathrm{I}}=U_{+} U_{-}$ and $U_{\mathrm{II}}=U_{-} U_{+}$with subscripts + and - denoting the travel paths with a positive or negative slope, respectively. ${ }^{11,19} \mathrm{Ow}-$ ing to the unitarity of the matrix $U$, one can easily perform the matrix product. We now seek to find the phase difference between the two paths, or equivalently, the phase factor that the traversing electron acquires upon circling the tilted square ring $U_{\text {phase }}=U_{\mathrm{II}}^{+} U_{\mathrm{I}}$. After some algebra, the eigenvalues of the phase factor $U_{\text {phase }}$ can be written as

$$
\lambda=1-2 q[1-\cos (2 \Delta \xi)] \pm i \sqrt{4 q(1-q)+q^{2} \sin ^{2}(2 \Delta \xi)},
$$

where $\quad q=\sin ^{2} \eta_{+} \sin ^{2} \eta_{-} \quad$ and $\tan (\Delta \xi)$ $=\left(\beta^{2}-\alpha^{2}\right) /[2 \alpha \beta \cos (2 \theta)] \quad$ with $\eta_{ \pm}=m \ell\left[\alpha^{2}+\beta^{2}\right.$ $\pm 2 \alpha \beta \sin (2 \theta)]^{1 / 2} / \hbar^{2}$. Alternatively, the eigenvalues can be expressed as $\exp ( \pm i \phi)$ since their modulus is unity. Thus, the phases acquired by the tilted up and down spins are $\pm \phi$. This shift is due to the SO coupling. One can check that for the normal square ring under pure Rashba coupling $(\beta=\theta=0$ and $\Delta \xi=-\pi / 2)$, the eigenvalues are $\lambda=1$ $-2 p \pm 2 i[p(1-p)]^{1 / 2}$ where $p=\sin ^{4} \eta$ with $\eta=m \ell \alpha / \hbar^{2}$. Setting $\phi=\pi / 2$ or $\lambda=1 / 2$, we obtain the same condition for achieving the ideal filters with Eq. (30) in Ref. 11, i.e., $\sin ^{4} \eta=1 / 2$, corresponding to the specific Rashba strength $\alpha^{*}=\left(\hbar^{2} / m \ell\right) \sin ^{-1}\left(2^{-1 / 4}\right)$.

To conclude, we have performed gauge transformation to derive the spin-rotation operator and the analytical expression of the spin precession for an ideally injected spin with arbitrary polarization under both the Rashba spin-orbit (RSO) and the Dresselhaus spin-orbit (DSO) couplings. The above two results are useful for analyzing the spatial behaviors of the RSP, the DSP, and the composite cases. In particular, the unique features of spin-precession patterns can be easily identified - aided by the spin-rotation axis, the precession angle, and the cone angle. We found that the RSP and DSP along general paths behave quite different; however, they exhibit similar behaviors along the specific directions $\phi= \pm 45^{\circ}$ and $\pm 135^{\circ}$. This implies that the spatial behaviors of spin precession, due to inversion asymmetry in the twodimensional electron gas (2DEG) along these four directions, are always invariant. Importantly, we explicitly pointed out that in the pattern of spin precession, there always exists a specific path with null spin precession and a special contour where the spins all return to its original orientation. We expect that these findings would facilitate to properly manipulate the spin polarization in spintronic devices based on exploiting the SIA and BIA effects.

\section{ACKNOWLEDGMENTS}

One of us (J.-S.Y.) acknowledges the support from National Science Council of the Republic of China under Grant No. NSC 96-2112-M-019-005. C.-R.C. thanks the National Science Council of the Republic of China for the support under Grant No. NSC 95-2112-M-002-044-MY3.

\footnotetext{
*crchang@phys.ntu.edu.tw

${ }^{1}$ Semiconductor Spintronics and Quantum Computation, edited by D. D. Awschalom, D. Loss, and N. Samarth (Springer, Berlin, 2002).

${ }^{2}$ I. Žutić, J. Fabian, and S. Das Sarma, Rev. Mod. Phys. 76, 323 (2004); E. I. Rashba, Physica E (Amsterdam) 34, 31 (2006).

${ }^{3}$ M.-H. Liu, Ching-Ray Chang, and Son-Hsien Chen, Phys. Rev. B 71, 153305 (2005).

${ }^{4}$ M. V. Costache, M. Zaffalon, and B. J. van Wees, Phys. Rev. B 74, 012412 (2006).
}

${ }^{5}$ E. I. Rashba, Sov. Phys. Solid State 2, 1109 (1960); Yu. A. Bychkov and E. I. Rashba, JETP Lett. 39, 78 (1984).

${ }^{6}$ J. Nitta, T. Akazaki, H. Takayanagi, and T. Enoki, Phys. Rev. Lett. 78, 1335 (1997); T. Koga, J. Nitta, T. Akazaki, and H. Takayanagi, ibid. 89, 046801 (2002).

${ }^{7}$ S. Datta and B. Das, Appl. Phys. Lett. 56, 665 (1990); M.-H. Liu and Ching-Ray Chang, Phys. Rev. B 73, 205301 (2006).

${ }^{8}$ G. Dresselhaus, Phys. Rev. 100, 580 (1955).

${ }^{9}$ E. A. de Andrada e Silva, Phys. Rev. B 46, 1921 (1992); S. D. Ganichev, V. V. Bel'kov, L. E. Golub, E. L. Ivchenko, P. 
Schneider, S. Giglberger, J. Eroms, J. De Boeck, G. Borghs, W. Wegscheider, D. Weiss, and W. Prettl, Phys. Rev. Lett. 92, 256601 (2004).

${ }^{10}$ David Z.-Y. Ting and X. Cartoixà, Phys. Rev. B 68, 235320 (2003).

${ }^{11}$ N. Hatano, R. Shirasaki, and H. Nakamura, Phys. Rev. A 75, 032107 (2007).

${ }^{12}$ J. Schliemann, J. C. Egues, and D. Loss, Phys. Rev. Lett. 90, 146801 (2003).

${ }^{13}$ X. Cartoixà, D. Z.-Y. Ting, and Y.-C. Chang, Appl. Phys. Lett. 83, 1462 (2003).

${ }^{14}$ For discussion about spin pumping in confined systems, see P.
Sharma, Science 307, 531 (2005).

${ }^{15}$ R. Winkler, Phys. Rev. B 69, 045317 (2004).

${ }^{16}$ B. A. Bernevig, J. Orenstein, and S. C. Zhang, Phys. Rev. Lett. 97, 236601 (2006).

${ }^{17}$ M.-H. Liu, K.-W. Chen, S.-H. Chen, and Ching-Ray Chang, Phys. Rev. B 74, 235322 (2006).

${ }^{18}$ P. Q. Jin, Y. Q. Li, and F. C. Zhang, J. Phys. A 39, 7115 (2006).

${ }^{19}$ Son-Hsien Chen and Ching-Ray Chang, Phys. Rev. B 77, 045324 (2008).

${ }^{20}$ J. J. Sakurai, Modern Quantum Mechanics, revised ed. (Addison-Wesley, New York, 1994). 